\title{
Research on a Reconfigurable Modular Manipulator System
}

(Report for Period June 15, 1989 - August 14, 1992)

\section{Project Investigators}

\author{
Professors Pradeep K. Khosla and Takeo Kanade
}

\author{
Address \\ Department of Electrical and Computer Engineering \\ and The Robotics Institute \\ Carnegie Mellon University \\ Pittsburgh, Pennsylvania 15213-3890
}

\section{DISCLAIMER}

\begin{abstract}
This report was prepared as an accoumt of work sponsored by an agency of the Urited States Governinent. Neither the United States Government nor any agency thereof, nor any of their employeses, makes any warranty, express or implied, or assumes any legal liability or responsibility for the accuracy, completeness, or usefulness of any information, apparatus, product, or process disclosed, or represents that its use would not infringe privately owned rights. Reference herein to any specific commercial product, process, or service by erade name, trademark, manufacturet, or otherwise does not necessarily constitute or imply its endorsement, recommendation, or favoring by the United States Government or any agency thereof. The views and opinions of authors expressed herein do not necessarily state of reflect those of the United States Government or any agency thereof.
\end{abstract}




\section{Description of Our Work during the Period June 89 - June 92}

\subsection{Introduction}

The technology for robotic manipulators has been developed primarily for industrial applications where the task environment can be engineered or constrained. Consequently, the dexterity and versatility of today's robotic manipulators are far from sufficien to perform tasks in an unpredictable, less constrained, and changing environment. One of the important reasons for this has been the lack of research in manipulator designs that embody the principles of reconfigurability and modularity, and the corresponding theories that allow the user to automatically map the task requirements into a kinematic configuration and the corresponding controller requirements. A thorough survey of researchers in the area of robotics identified the importance and need for reconfigurability and modularity in manipulator designs in order to enhance their versatility.

Presently a specific manipulator is chosen for a specific task and if the task requirements were to change a different manipulator would be required. In dynamic environments that may be ncountered in a nuclear facility or aboard a space station it is desirable to have a manipulator system that could, within reason, reconfigure itself and adapt to the new task requirements. For example, consider the use of manipulators in a field or in a nuclear facility that must assemble, and repair large and small structures (e.g. antenna towers, trusses) and equipment (e.g. vehicles, electronics). In such an environment, oftentimes widely varied kinematic (e.g., workspace) and dynamic (e.g., speed, accuracy) performance is desired. Generally, either no single conventional manipulator is capable of delivering these requirements, or it is too expensive to prepare a large set of manipulators to accomplish these tasks. Moreover in some other situations, the manipulator kinematics and dynamics requirements may not be even known a priori, and yet a manipulator is to be deployed for performing the task.Thus, there exists a need for advanced manipulators that can be rapidly reconfigured to cover a wide range of task requirements.

To fulfill this need, we have been pursuing research, under a grant from DOE Office of Basic Energy Sciences for the period June 1989- August 1992, on developing the theoretical basis and the technology for Reconfigurable Modular Manipulation System (RMMS). Unlike a conventional manipulator which has a fixed configuration, the RMMS consists of a set of interchangeable modules that can be rapidly assembled into a system of manipulators with appropriate configurations depending on the specific task requirement. For effective development and use of such a versatile and flexible system we have been pursuing a program of theoretical and experimental research aimed at developing the basis for next generation of autonomous manipulator systems. The RMMS concept exterids the idea of autonomy from sensor-based to configuration based autonomy. One of the important components of our research is the development of design methodologies for mapping tasks into manipulator configurations and for automatic generation of manipulator specific algorithms 
(e.g., kinematics and dynamics) in order to make the hardware transparent to the user.

During this period of performance we have made significant progress towards accomplishing both the theoretical and experimental objectives of our grant. In the next section, we describe our accomplishments in further detail. In Section 1.2.1. we provide a detailed list of the publications and invention disclosures that have resulted from our research.

Our research has not only made contributions to the theory of reconfigurable manipulator systems but it has also impacted robotics in DOE. Based on our work, DOE has included modular and reconfigurable systems, in their 5 year plan for the ER\&WM (Environmental Restoration and Waste Management) program, as one of the key technologies that must be developed. In the following sections, we describe our accomplishments under the current grant.

\subsection{Accomplishments during the Previous Grant Period}

In order to provide a context for our contributions, we first describe the work that was proposed in our previous proposal which was funded for a three year period beginning June 15 , 1989. We would like to emphasize that we not only completed the promised work but also extended it in ways that has lead to the generation of new ideas (such as, design of reliable manipulators and the use of genetic algorithms for design of manipulators). Our past statement of work included the following tasks:

Configuring a Manipulator from Task Requirements: In this part of our research, we will develop methodologies that map the task requirements into a specific manipulator. The kine. matic task requirements will be used to determine the link lengths and the orientation of the modules. And the dynamic task requirements will be translated to obtain the sizes and ratings of the actuators or joints. We will investigate the use of both rule-based expert systems and optimization techniques in obtaining a solution to this problem.

Automated Generation of Kinematics: In order to obtain a mapping from the task to joint coordinates and vice-versa, we will develop techniques to automatically determine the kinematics of modular manipulators. Specifically, we will obtain the forward and the inverse kinematics and the Jacobian transformations for modular manipulators. Since redundancy is an important property of modular manipulators, we will also concentrate on inverse kinematics for redundant manipulators.

Experimental Demonstration: In this part of our research, we will experimentally demonstrate the theoretical concepts. We will construct experimental prototype modules of the RMMS for this purpose. We will choose a set of representative tasks to which our methodology will be applied to obtain the manipulator configuration. We will then manually assemble the manipulator and apply the manipulator to accomplish the task. (It must be noted that the ultimate goal is to automatically assemble the manipulator but this task is presently out of the scope of the proposed research). Our techniques for automatically generating the kinematics and the reconfigurable controllers will be demonstrated in the execution of the task. 
Our accomplishments during the past period span both theoretical and experimental contributions. In the following paragraphs we only provide a brief synopsis of our contributions.

Specifically, on the theoretical side, we have developed:

\section{Methodologies for kinematic design of manipulators from task specifications.}

We have developed methodologies that take the kinematic task characteristics as input and create a manipulator configuration (or Denavit-Hartenberg parameters of the manipulator) as output. We define tasks with various aitributes such as reachability (or workspace), manipulability (or the ability to reach a point in a good posture). We also include constraints such as joint limits, and obstacles in the workspace. We have shown that for 2 DOF planar manipulators there exists an analytical solution to determine the manipulator configuration. We use hyperplanes in kinematic space to develop an analytical solution for determining the kinematic configuration of the manipulator. In our approach, we use Denavit-Hartenberg parameters to specify the kinematic configuration.

Since deriving analytical solutions for arbitrary spatial manipulators is extremely difficult, if not impossible, we developed a numerical procedure for the design of 3-D spatial systems. In order to accomplish our objective, we posed the kinematic design problem as an optimization problem. Our approach is different from tradi. tional approaches in kinematic synthesis of manipulators. One of the main differences is that most of these approaches address the problem of determining the link lengths only. In contrast, our approach addresses the problem of determining the full kinematics that include the link lengths and also the twist angles. Another difference is that previous approaches concentrated only on the workspace characteristics. In contrast, our approach looks at the task characteristics, such as workspace, manipulability, and obstacles, and it also takes into account the physical limitations of the manipulator, such as joint limits. Our formulation is general and allows the incorporation of other task characteristics, such as accuracy, force application capability, etc.

We have implemented simulated annealing as the optimization procedure. We have also used the numerical formulation to check the validity of the 2 DOF analytical solutions and to show how 6 DOF spatial manipulators can be designed using our approach. It is interesting to note that our solution process results in both intuitive and non-intuitive solutions for 3-D spatial manipulators.

The above work accomplishes design of the kinematics of the manipulator in continuous space. That is, the Denavit-Hartenberg parameters can vary in a continuous manner. However, for RMMS design we need a method that accomplishes design in discrete space because the components of an RMMS are available in discrete values. For example, two adjacent modules can have only 16 possible twist angles between them and links are available in a finite number of discrete lengths.

In order to accomplish discrete design, we proposed and developed a design meth- 
odology based on genetic algorithms. Since traditional genetic algorithms are not suitable for our problem, we developed a Multi-Population Genetic Algorithm (MPGA) in order to accomplish our objective. Furthermore, we were able to develop algorithms that could synthesize both the kinematic configuration and the base location (of the manipulator) simultaneously. Design of base locations is important because in unstructured environments, it is not always possible to determine where the base of the manipulator must be placed to accomplish the task. Our work on including the bast position as a design parameter is preliminary and limited to planar systems. We propose to continue this line of investigation in this renewal proposal.

\section{Methodologies for dynamic design of manipulators from task specifications.}

Besides determining the kinematic parameters, it is also important to develop synthesis methodologies that determine the dynamic parameters from task requirements. For a N DOF manipulator, it is necessary to determine the $10 \mathrm{~N}$ parameters (10 parameters per link) that describe the dynamic behavior of the system. We have developed an algorithm that takes the dynamic characteristics of the task as input and creates as its output the modules of the RMMS that must be used to achieve the desired dynamic behavior. The dynamic characteristics of the task are described in terms of desired maximum velocities and accelerations, and the desired payload carrying capability. The kinematic structure (determined from the kinematic design process) and the dynamic parameters of the individual RMMS modules (such as maximum torque rating) are used as additional input to our design process.

Our solution technique first translates the design problem into a tree search problem. We then use a recursive search method with backtracking to search the tree for the appropriate modules. We use nonlinear optimization to ensure that the maximum torque rating at any joint can be obtained from the actuator module. In order to reduce the computational requirements, we first prune the tree on the basis of simplified dynamic equations. In the second step we use the complete dynamics formulation to ascertain that the task requirements are met. We have implemented this algorithm and used it to determine the RMMS modules that must be used for various task specifications.

3. Techniques for automatically generating the kinematics and dynamics for arbitrary manipulators created from the RMMS modules.

Since arbitrary manipulators can be configured for a specific task (based on the design algorithms that we have developed), it is important to develop techniques that will automatically generate the kinematic and dynamics of this arbitrarily configured manipulator. We would like to emphasize that this problem is not the same as developing kinematics and dynamics symbolically.

Each module of the RMMS (both joint and link) is totally integrated and contains a microprocessor or ROM that stores that kinematic and dynamic parameters of that 
module. When two modules are connected a series of LED's and photodetectors (located at the mechanical interface) determine the relative orientation of the adjacent modules. Every module then transmits its parameters over the communication and control bus to the host computer. We have developed algorithms that accept this information and develop the forward and inverse kinematics, and the dynamics description of the configured manipulator. The automatic generation of kinematics and dynamics makes the hardware and its structure completely transparent to the user. Therefore, the user can write device independent and task dependent code. In some sense automatic generation of kinematics and dynamics plays the role of a compiler since the detailed structure of the manipulator is not required to be known by the user to write applications code.

Since it is possible to configure a redundant manipulator, our algorithm generates the kinematics numerically for both redundant and non-redundant manipulators. We have implemented our techniques on the experimental RMMS that we have built and demonstrated their effectiveness in the context of accomplishing a variety of tasks.

4. A real-time control architecture for controlling arbitrary manipulators cre. ated from RMMS modules.

In order to control arbitrary manipulators created from RMMS modules, we have also developed a real-time control architecture. This architecture is based on commercially available SUN host and Ironics $680 \mathrm{xx}$ based CPU's. Our architecture supports multiple CPU's and I/O devices that may be connected to the configured RMMS. We have also developed a real-time operating system CHIMERA II (supported mainly by DARPA) that runs on the real-time controller architecture.

We would like to emphasize that this architecture is general purpose and is used to control a variety of manipulators in our laboratory including the CMU Direct Drive Arm II, the RMMS, multiple PUMA 560 manipulators, and the Space manipulator system. Further, we have also been successful in transferring the CHIMERA II real-time system and the real-time architecture to JPL where it is currently being used to control the redundant Robotics Research Arm (under a hierarchical control structure).

5. A unified representation for controlling redundant manipulators that may be created from RMMS modules.

Since a manipulator configured from RMMS may be redundant, it is important to develop methodologies for controlling redundant manipulators. A lot of work has been done by several researchers for controlling redundant manipulators. Our work rmphasizes the development of unified representations and models that allow us to implement any of the chosen control algorithms. We have proposed the Premultiplier model for the development of this unified representation. Our representation provides both insight and new results. For example, we show how the decomposed and decoupled static behavior of redundant manipulators can be used 
to derive an optimal control strategy.

Besides pursuing theoretical research, we have also been involved in demonstrating our ideas experimentally. Specifically,

1. We have built two types of prototype joints: Rotate and Pivot

2. We have built 3 Pivot joints and 1 Rotate joints and constructed a prototype RMMS.

3. We have built a new type of coupling mechanism to connect the modules of the RMMS. We have filed an invention disclosure for a possible patent based on this work.

4. We have built the electronics for a novel bus called the Armbus. This bus runs throughout the RMMS and every module of the RMMS is a logical element on this bus. This bus is mainly used for control and communications functions. The novelty of this bus is that it removes the need to run cables, for power and data, through the RMMS.

5. We have implemented our design methodologies (for kinematic and dynamic synthesis) and used them to generate specific configurations of manipulators. We have also built these manipulator configurations (from the available RMMS modules) and used them to show that they satisfy the task requirements that the system was designed for.

In the next section, we provide a list of publications and invention disclosures that have resulted from our work during the performance period.

\subsubsection{List of Patents/Invention Disclosures}

Our research has resulted in the following invention disclosure to $\mathrm{CMU}$ for a possible patent.

1. Eric Hoffman, Mark DeLouis, and Pradeep K.Khosla, A Robotic Manipulator Joint/Link Mechanical Coupler, Invention Disclosure filed with Office of Technology Transfer, Carnegie Mellon University, Ref Number 92-008, September, 1991.

\subsubsection{List of Papers Related to the Grant}

Our research has resulted in the following publications:

1. Khosla, P. K., Kanade, T., Schmitz, D., Hoffman, R., and Delouis, M., The CMU Reconfigurable Modular Manipulator System, RI Research Review, 1989.

2. Pareedis, C. J., and Khosla, P. K., An Approach for Mapping Kinematic Task Specifications into a Manipulator Design, Fifth International Conference on Advanced 
RoboAdvanced Robotics, Pisa, Italy, June, 1991. An extended version of this paper titled "Task Based Design of Manipulator Kinematic Configurations" has been submitted to International Journal of Robotics Research, MIT Press.

3. Pareedis, C., J., and Khosla, P. K., On Kinematic Design of Serial Link Manipulators, Proceedings of International Symposium on Intelligent Robotics, Bangalore, India, January, 1991.

4. Kelmar, L., and Khosla, P. K., Automatic Generation of Kinematics for a Reconfigurable Modular Manipulator System, Journal of Robotic Systems, August 1990.

5. Krishnan, A., and Khosla, P. K., A Methodology for Determining the Dynamic Configuration of a Reconfigurable Modular Manipulator System, Proceedings of International Symposium on Measurement and Control in Robotics, Houston, Texas, June 1990. (Also being revised for Journal of Robotic Systems).

6. Kim J-O., Khosla, P. K., and Chung, W. K., Static Modeling and Control of Redundant Manipulators, Robotics and Computer Integrated Manufacturing, Pergamon Press, Volume 9, Number 2, 1992.

7. Kim, J-O, and Khosla, P. K., Dexterity Measures for Design and Control of Manip. ulators, Proceedings of IEEE International Workshop on Intelligent Robot Systems, Osaka, Japan, November, 1991.

8. Kim, J-O, and Khosla, P. K., A Multi Population Genetic Algorithm and its Application to Design of Manipulators, Proceedings 1992 IROS Conference, Raleigh, North Carclina.

9. Kim, J-O., and Khosla, P. K., Task-Based Design of Manipulators, In Proceedings of 1992 ISRAM, Albuquerque, June, 1992. (accepted for publication).

10. Schmitz, D., Khosla, P. K., and Kanade, T., The CMU Reconfigurable Modular Manipulator System, Proceedings of 19-th International Symposium on Intelligent Robotics, Australia, November, 1989.

Besides the above publications, the RMMS has also been featured on the cover of ASME Mechanical Engineering Magazine and Production magazine during 1991.

\subsubsection{MS/PhD Thesis Supported under the Grant}

Our current grant includes support for 1.5 graduate students per year. During the period of the grant, we have been able to recruit some students who were partially supported by fellowships. This has allowed us to leverage the grant monies and therefore accomplish more work than would have been possible. The following work was fully or partially supported by the grant.

Arun Krishnan, A Methodology to Determine the Dynamic Configuration of a Reconfig- 
urable Modular Manipulator System, MS Thesis (completed).

Laura Kelmar, Automatic Generation of Kinematics for a Reconfigurable Manipulator System, MS Thesis (completed).

Christaan Pareedis, An Approach for Mapping Kinematic Task Specifications into a Manipulator Design. MS Thesis (complated).

Christaan Pareedis, Presently Pursuing a PhD degree, Expected Completion date for the PhD is January 1995.)

Jin-Oh Kim, Task Based Design of Manipulators. PhD thesis (expected to finish by February 1993).

Fred Au, Design of Redundant and Reliable Manipulators, MS Thesis, May, 1992. 

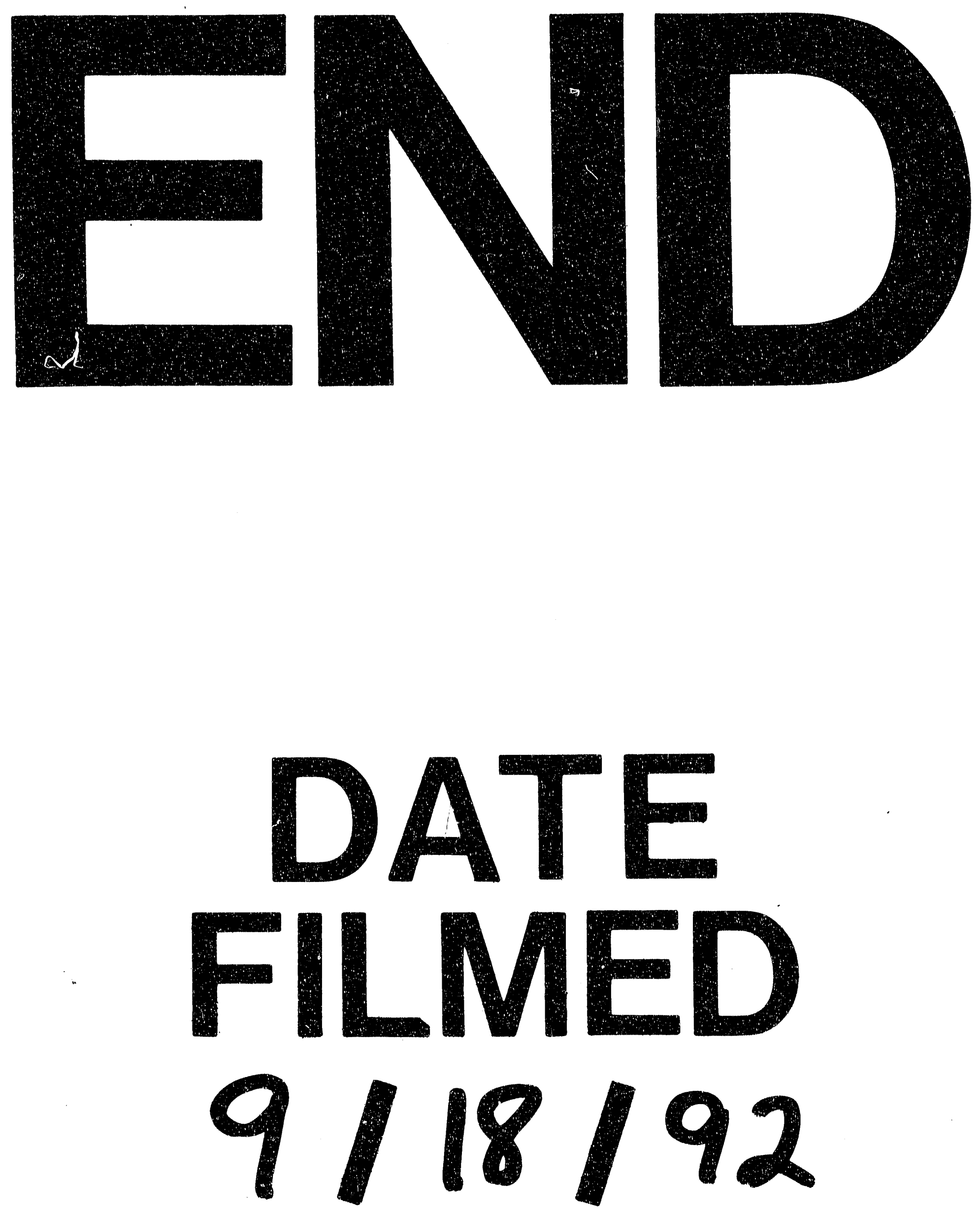
